\title{
Assessment of markers expressed in human hair follicles according to different skin regions
}

\author{
Arzu Yay ${ }^{1, A-E}$, Özge Göktepe ${ }^{1, B, C}$, Anzel Bahadir ${ }^{2, D, E}$, Saim Özdamar ${ }^{1, A}$, Ibrahim Suat Öktem ${ }^{3, B}$, Atilla Çoruh ${ }^{4, B}$, Münevver Baran ${ }^{5, C, D}$ \\ 1 Department of Histology and Embryology, Faculty of Medicine, Erciyes University, Kayseri, Turkey \\ ${ }^{2}$ Department of Biophysics, Faculty of Medicine, Düzce University, Turkey \\ ${ }^{3}$ Department of Neurosurgery, Faculty of Medicine, Erciyes University, Kayseri, Turkey \\ ${ }^{4}$ Department of Plastic and Reconstructive Surgery, Faculty of Medicine, Erciyes University, Kayseri, Turkey \\ ${ }^{5}$ Department of Pharmaceutical Basic Science, Faculty of Pharmacy, Erciyes University, Kayseri, Turkey \\ A - research concept and design; $\mathrm{B}$ - collection and/or assembly of data; $\mathrm{C}$ - data analysis and interpretation; \\ $D$ - writing the article; $E$ - critical revision of the article; $F$ - final approval of the article
}

\author{
Address for correspondence \\ Arzu Yay \\ E-mail: arzu.yay38@gmail.com \\ Funding sources \\ None declared \\ Conflict of interest \\ None declared

\section{Acknowledgements} \\ This work was supported by the Scientific \\ Research Project Coordination Unit of Erciyes \\ University (Project number: EUBAP, TCD-4517).
}

Received on February 4, 2017

Reviewed on May 23, 2017

Accepted on June 5, 2017

DOI

10.17219/acem/74429

\section{Copyright}

Copyright by Author(s)

This is an article distributed under the terms of the

Creative Commons Attribution Non-Commercial License

(http://creativecommons.org/licenses/by-nc-nd/4.0/)

\section{Abstract}

Background. Body region-dependent hair follicle (HF) characteristics are concerned with follicular size and distribution, and have been demonstrated to have characteristics for each region of the body.

Objectives. The aim of the present study was to investigate the expression patterns of the markers called cytokeratin 15 (K15), cytokeratin 6 (K6) and monoclonal antibody Ki-67, and also apoptosis in HFs, which can be observed in different parts of the human body.

Material and methods. In this study, healthy human HFs were taken by biopsy from 5 various donor sites of the human body: the scalp, the leg, the abdomen, the back and waist. HF-containing skin specimens taken using cryosection were stained with hematoxylin \& eosin (H\&E) and K15, K6, Ki-67 and terminal deoxynucleotidyl transferase-mediated digoxigenin-dNTP nick end-labelling (TUNEL) immunofluorescence staining protocol was performed.

Results. Different skin regions from the human body were examined histologically. While the HFs of scalp tissue showed anatomically obvious hair layers, some hair sections from other regions, like the leg, the abdomen, back and waist, were not as distinct as in the scalp region. According to our findings, K15 expression was highest in the scalp. In addition, the immunoreactivity (IR) intensity of K15 was significantly decreased in the HFs on the waist and abdominal regions, compared to the scalp and back regions ( $p<0.001)$. However, the IR intensity of K6 in the scalp region was statistically significantly higher than the IR intensity of K6 in the abdomen region $(p<0.05)$. Moreover, we showed intraepithelial apoptosis and proliferation of keratinocytes in the bulge of HF. In the study, Ki-67-positive and TUNEL-positive cell numbers were not statistically significant $(p>0.05)$.

Conclusions. Our findings are important for further investigation of molecular aspects of the human hair follicle stem cells compartments in health and disease, which might be a promising model for comparative studies with different human diseases.

Key words: monoclonal antibody Ki-67, terminal deoxynucleotidyl transferase-mediated digoxigenin-dNTP nick end-labelling, hair follicle, cytokeratin 15, cytokeratin 6 


\section{Introduction}

Hair follicles (HFs) as a functional mini-organ, form a very complex structure which consists of mesenchymederived dermal papilla and 3 distinct epithelial layers: the outer root sheath (ORS) (the outer layer), the inner root sheath (IRS) (the middle layer) and the hair shaft (the central layer). ${ }^{1}$ The mammalian hair follicle contains a heterogeneous cell population, including hair follicle stem cells (HFSCs) and epithelial cells, such as keratinocytes. ${ }^{2}$ The diversity of the morphologic differentiation pathway of the hair follicle is partly reflected by the respective keratin protein patterns. The HF keratin patterns are mainly composed of the epithelial keratins. ${ }^{3}$ These keratins belong to a heterogeneous family of intermediate filaments that are essential to the epidermis, where they are expressed in a cell-type and differentiation-specific manner during epithelial development and differentiation. ${ }^{4,5}$ In humans, keratins generally present in epithelial cells as keratinocytes. ${ }^{6}$ The main function of keratins is the maintenance of cell and tissue structure. ${ }^{5,7}$ Expression of specific keratins and changes in the expression profile temporally and spatially reflect the differentiation status of epidermal cells both in development and in mature epidermal tissue. ${ }^{8}$

The expression of cytokeratin 15 (K15) is thought to have stem cell characteristics ${ }^{9,10}$ and enrichment has been observed in the HF bulge region where multipotential epidermal stem cells are located. ${ }^{11,12}$ The HFSCs sustain growth and cycling of the HF and they are known to play a major role in maintaining skin homeostasis. ${ }^{13}$ Actually, human HF bulge is an important niche for HFSCs and several authors have described K15 as a putative epidermal stem cell marker. ${ }^{12,14,15}$ In humans, cytokeratin 6 (K6), which is expressed in epithelial appendages, has distinct tissue expression patterns, and it is the dominant form in epithelial tissue. $\mathrm{K} 6$ is expressed in the inner bulge layer and is implicated in maintaining HFSC quiescence, whereas strong $\mathrm{K} 6$ expression was observed in the interfollicular epidermis but not in the bulge region. ${ }^{16}$ The expression of the human Ki-67 protein is properly associated with cell proliferation and this makes it an excellent marker for determining the growth fraction of a given cell population. ${ }^{17}$ Indeed, Ki-67 immunoreactivity (IR) is a useful tool for cell proliferation in HF matrix keratinocytes below the widest part of the dermal papilla and bulge. The onset of catagen is characterized by a noticeable reduction in the percentage of Ki-67-positive matrix keratinocytes, with virtually no Ki-67-positive HF keratinocytes present in late catagen. ${ }^{18}$

Over the years, both human and mouse disorders have shown additional complex functional roles for cytoskeleton keratins, which are involved in the modulation of apoptosis, cell growth, tissue polarity, wound response and tissue remodeling. 5,7,19 The HF is an established tissue source for cell-based therapy. The skin stem cells of HF are in clinical set up for a long period of time and many cell-based applications are there for the management of disorders. Thus, some investigators have used HFSCs for cell-based clinical needs such as vitiligo. ${ }^{20}$ In this context, the results of many studies have suggested that an abnormal stem cell marker, such as K15 expression, potentially plays a role in the pathogenesis. ${ }^{21}$

Previous studies have shown body region-dependent HF characteristics concerning follicular size and follicular distribution, and demonstrated that each region of the body disposes of its own hair follicle characteristics. In these studies, the hair follicle density on the forehead, cheek, chest and back of the human body has been determined. ${ }^{22}$ Nevertheless, the expression patterns of useful markers used in HF studies in different skin regions are poorly understood. However, the determination of these markers in different skin regions and a comparison of expression intensities can be an important contribution to treatments for skin injury, cancers and hair loss. Hence, we aimed in this preliminary study to evaluate the expression intensities of markers such as K15 and K6 identified in hair follicle cells found in different skin regions. Moreover, to the best of our knowledge, this is the $1^{\text {st }}$ study to evaluate apoptosis and proliferation of keratinocytes in the HF bulge of different skin regions.

\section{Material and methods}

The study was approved by the clinical research ethics committee of Faculty of Medicine, Erciyes University, Kayseri, Turkey (number: 197). All volunteers gave written informed consent and the protocol was approved by the institutional review board.

\section{Specimens}

Human healthy hair skin biopsy specimens were taken from each routine surgery from 5 various donor sites (scalp, leg, abdomen, back and waist). This process was conducted with written consent, under the same conditions, i.e., at the same room temperature and humidity. The voluntary donors were all patients from the Neurosurgery Department and the Plastic and Reconstructive Surgery Department at the Erciyes University, Kayseri, Turkey. All experiments were carried out according to the Helsinki guidelines, in compliance with national regulations for the experimental use of human material. We examined 38 samples of healthy skin from various donor sites and their description is shown in Table 1. None of the volunteers suffered from any kind of skin disease, hormonal dysregulation or adiposity. Also, they did not have any diseases such as cancer or autoimmune diseases. The excised hair skin biopsies were transported in Williams E medium (Biochrom, Cambridge, UK) on ice and transferred to the laboratory of histology and embryology immediately. 
Table 1. Classifications of healthy skin specimens gathered from various donor sites of the body according to individual's gender and age range

\begin{tabular}{|l|c|c|c|c|}
$\begin{array}{c}\text { Body } \\
\text { region }\end{array}$ & $\begin{array}{c}\text { Female } \\
{[\mathrm{n}]}\end{array}$ & $\begin{array}{c}\text { Male } \\
{[\mathrm{n}]}\end{array}$ & $\begin{array}{c}\text { Total } \\
{[\mathrm{n}]}\end{array}$ & $\begin{array}{c}\text { Age range } \\
\text { [years] }\end{array}$ \\
\hline Scalp & 6 & 5 & 11 & $22-60$ \\
\hline Leg & 3 & 10 & 13 & $24-72$ \\
\hline Back & 6 & 4 & 10 & $30-75$ \\
\hline Abdomen & 5 & 3 & 8 & $31-69$ \\
\hline Waist & 5 & 4 & 9 & $29-58$ \\
\hline
\end{tabular}

$\mathrm{n}$ - number of examined healthy individuals.

\section{Tissue preparation and histochemistry analysis}

Normal hair skin tissue specimens were first shaved using a scalpel and dissected into approx. $0.5 \mathrm{~cm}^{2}$ pieces before being washed 1-3 times with phosphate-buffered saline (PBS) to remove cell debris. The skin biopsies were embedded in Shandon Cryomatrix (Thermo Fisher Scientific, Waltham, USA), snap-frozen in liquid nitrogen, and stored at $-80^{\circ} \mathrm{C}$ until use. All specimens were covered with poly-L-lysine and the sections were placed on slides for examination. Cryosections ( $7 \mu \mathrm{m}$ thick) were first air-dried for $10 \mathrm{~min}$, fixed in acetone at $-20^{\circ} \mathrm{C}$ for $10 \mathrm{~min}$, and then air-dried again for $10 \mathrm{~min}$. Longitudinally cut hair skin specimens were stained by hematoxylin \& eosin (H\&E). The hair skin sections were studied using light microscopy (Olympus BX51 fluorescent microscope; Olympus, Tokyo, Japan). Photos were taken using a Olympus DP71 (Olympus, Tokyo, Japan) digital camera.

\section{Immunofluorescence staining}

Immunofluorescence staining was used to detect K15, K6, Ki-67 and terminal deoxynucleotidyl transferase-mediated digoxigenin-dNTP nick end-labelling (TUNEL) IRs in the HFs.

\section{K15 immunofluorescence staining}

To identify the IR of K15 in the bulge region of the HFs in human skin via immunofluorescence staining, we used the tyramide signal amplification method, according to Kloepper et al. ${ }^{23}$ After fixation, the cryosections were washed 3 times by using a TNT (Tris- $\mathrm{HCl} \mathrm{NaCl}$ Tween) buffer for $5 \mathrm{~min}$. Next, horseradish peroxidase was blocked by washing with $3 \% \mathrm{H}_{2} \mathrm{O}_{2}$ in PBS for $15 \mathrm{~min}$. The sections were incubated with avidin and biotin for $15 \mathrm{~min}$. Preincubation was performed in $5 \%$ goat normal serum in TNT for $30 \mathrm{~min}$. Primary antibodies (K15) diluted in TNT (1:400 dilution Chemicon CBL; Merck, Billerica, USA) were incubated overnight at $4^{\circ} \mathrm{C}$, followed by a biotinylated secondary antibody goat anti-mouse (1:200 dilution in TNT) for $45 \mathrm{~min}$ at room temperature (RT). For the next steps, a tyramide signal amplification kit (TSA kit; Perkin-Elmer,
Boston, USA) was administered (1:100 dilution in TNT) for $30 \mathrm{~min}$ at RT. The reaction was amplified by tetramethylrhodamine-tyramide amplification reagent at RT for $5 \mathrm{~min}$. The cryosections were counterstained with 4;6-diamidino2-phenylindole (DAPI) (Sigma, St. Louis, USA) for nuclear staining for $1 \mathrm{~min}$ and mounted with Fluoromount-G (Southern Biotechnologies, Birmingham, USA).

\section{K6 immunofluorescence staining}

Cryosections $(7 \mu \mathrm{m})$ stored at $-80^{\circ} \mathrm{C}$ were first air-dried for $10 \mathrm{~min}$, fixed in acetone at $-20^{\circ} \mathrm{C}$ for $10 \mathrm{~min}$ and then air-dried again for $10 \mathrm{~min}$ at RT. After the slides were washed 3 times for 5 min with tris-buffered saline (TBS), they were preincubated with normal goat serum $(10 \%$ in trisbuffered saline) for 20 min. Primary antibody K6 (1:400 in TBS) (Progen, Heidelberg, Germany) was applied directly on the sections and the slides were incubated overnight at $4^{\circ} \mathrm{C}$ in a humidity chamber. After washing 3 times for $5 \mathrm{~min}$ in TBS, the sections were stained with goat antimouse secondary antibody and conjugated with fluorescein isothiocyanate $(\mathrm{FITCH})$ (1:200 dilution in TBS; Jackson ImmunoResearch, Cambridgeshire, UK) for $45 \mathrm{~min}$ at RT. The slides were again washed 3 times for $5 \mathrm{~min}$ in TBS. Following each step, the cryosections were counterstained with DAPI (Sigma, St. Louis, USA) for $1 \mathrm{~min}$. The slides were mounted by using Fluoromount-G (Southern Biotechnologies, Birmingham, USA). For immunostaining assays, primary antibodies were omitted as a negative control.

\section{Ki-67 and TUNEL double staining assay}

To compare proliferation and apoptosis of human HFs in the various hair cycles of different body regions, $\mathrm{Ki}-67$ and in situ terminal deoxynucleotidyl transferasemediated digoxigenin-dNTP nick end-labelling (TUNEL) double staining method were used. The TUNEL method was utilized to show the apoptotic cells of human HF, while the Ki-67 marker was used for detecting proliferating cells. For double immunofluorescence staining of TUNELpositive cells and Ki-67 IR, the protocol for the TUNEL technique was combined with the manufacturer's protocol for Ki-67 immunofluorescence. ${ }^{24}$ The TUNEL apoptosis detection kit (ApopTag Fluorescein in Situ Apoptosis detection kit; Millipore, Berlin, Germany) was used according to the manufacturer's protocol. TUNEL staining was performed using standard protocols. Briefly, $7 \mu \mathrm{m}$ frozen tissue sections were air-dried for $15 \mathrm{~min}$ and postfixed in ethanol-acetic acid $\left(-20^{\circ} \mathrm{C}\right)$ for $5 \mathrm{~min}$, followed by incubation with digoxigenin-dUTP in the presence of TdT (terminal deoxynucleotidyl transferase) for $1 \mathrm{~h}$ at $37^{\circ} \mathrm{C}$. TUNEL-positive cells were visualized by anti-digoxigenin fluorescence unconjugated antibody. Subsequently, the sections were pre-saturated with normal goat serum and incubated with the primary Ki-67 antibody (mouse anti-human 1:100 in PBS) (DAKO, Carpinteria, USA) overnight 
at $4^{\circ} \mathrm{C}$. The cryosections were incubated with a secondary rhodamine-labelled goat anti-mouse antibody (1:200 dilution in PBS; Jackson ImmunoResearch, Newmarket, UK) for $45 \mathrm{~min}$ at RT after a washing step. Nuclear counter staining was performed with DAPI (Sigma, St. Louis, USA) to stain the cell nuclei and mounted with Fluoromount-G (Southern Biotechnologies, Birmingham, USA). Negative controls were performed by omitting TdT and the Ki-67 antibody. Images were visualized under an Olympus BX51 fluorescence microscope (Olympus, Tokyo, Japan).

\section{Histomorphometry and statistical analysis}

The immunostaining intensity levels for the selected examined antigens were compared by quantitative immunohistochemistry using Image J software (ImageJ, National Institute of Health, USA). The IR intensities of K15 and K6 of at least 3 HFs randomly defined for 5 different groups were evaluated from each individual. The mean of the IR intensities for K15 and K6 was calculated by using Image J software at high power fields ( $\times 400$ magnification). To obtain numbers for Ki-67 and TUNEL-positive cells, 3-7 hair follicles per each region were analyzed. The numbers of the Ki-67 and TUNELpositive cells in the bulge regions of each HF were counted by Image J software, irrespective of the follicular cycle.

For evaluating statistical significance, these measurements were pooled and the mean \pm standard deviation values were calculated. All statistical procedures were performed by statistical analysis software SPSS v. 15.0 (SPSS Inc., Chicago, USA). One-way analysis of variance (ANOVA) compared the staining intensity values between groups. When the p-value from one-way ANOVA test statistics was statistically significant, a post hoc Tukey HSD nonparametric multiple comparison test was used to determine which group differed from which others. The results were considered significant if the $\mathrm{p}$-value was $<0.05$.

\section{Results}

\section{Histological findings}

A comparison of HF samples originating from different donor sites showed the same features as those known from the histologic examination. In the study, primarily the histological structures of HFs were examined in hairy skin specimens taken from different regions of the human body, and the HFs were evaluated histologically. Staining the HF sections with H\&E allows a clear visualization of the different follicular compartments. Histological images from HFs of different skin regions are shown in Fig. 1. From the histological examination, it was observed that HFs from scalp tissue showed a complete histological stratification, whereas HFs from other regions (leg, abdomen, back and waist) did not show complete stratification.

\section{Immunofluorescence findings}

In this part of the study, K15, K6, Ki-67 and TUNEL immunofluorescence staining used in HF hair follicle studies were selected and expression intensities in the HFs from different skin regions of these antibodies were calculated. K15-positive IR was observed in the outermost layer of the outer root sheath in the bulge of HFs, corresponding to the human bulge region in all regions of HFs. When the HFSCs with K15 IR intensities were compared with other regions, it was observed that the highest expression was in the HFSCs taken from the scalp tissue. Interestingly, when K15 expression was evaluated, the HFSCs in the back and leg region showed almost as much as the HFSCs in the scalp (Table 2). In our study, the decrease in IR intensity of K15 was statistically significant in the HFs on the waist and abdominal regions, compared to the scalp and back regions $(\mathrm{p}<0.001)$. Additionally, there were no significant differences between in the HFs taken from the scalp region and the back or leg regions in terms of K15 IR intensity ( $\mathrm{p}>0.05$ ) (Fig. 2).

Moreover, K6 expression was present in the keratinocyte cells of all HFs when the HFs in different skin regions were evaluated in terms of $\mathrm{K} 6$ expression levels. However, it was determined that the IR intensity of K6 differed according to the region of the HF. The HFs with the most intensive $\mathrm{K} 6$ expression was observed in the scalp tissue. Immunoreactivity intensity of $\mathrm{K} 6$ was higher in the scalp region than the abdomen region, and this difference was statistically significant $(\mathrm{p}<0.05)$. According to our results, while the HFs in the back region showed similar K6 expression to the scalp HFs, K6 levels were not statistically significantly different between the scalp and back regions, as shown in Table 2 ( $\mathrm{p}>0.05$ ) (Fig. 3).

Table 2. Immunoreactivity intensity of K15, K6 and Ki-67 terminal deoxynucleotidyl transferase-mediated digoxigenin-dNTP nick end-labelling (TUNEL)-positive cell number in HFs of different skin regions

\begin{tabular}{|l|c|c|c|c|c|c|}
\hline \multicolumn{1}{|c|}{ Markers } & Scalp & Leg & Abdomen & Back & Waist \\
\hline K6 & $74.67 \pm 13.35^{*}$ & $70.90 \pm 11.12$ & $68.63 \pm 16.99$ & $73.35 \pm 13.33$ & $70.34 \pm 11.01$ \\
\hline Ki-67 & $17.85 \pm 6.92$ & $15.47 \pm 5.77$ & $14.67 \pm 5.08$ & $14.67 \pm 5.08$ & 0.020 \\
\hline K15 & $38.58 \pm 9.80^{* *}$ & $33.17 \pm 8.43$ & $30.88 \pm 8.52$ & $35.27 \pm 8.57^{* *}$ & $32.73 \pm 7.91$ \\
\hline TUNEL & $6.93 \pm 3.50$ & $6.31 \pm 3.35$ & $6.33 \pm 3.55$ & $6.23 \pm 4.00$ & $<0.001$ \\
\hline
\end{tabular}

Data is expressed as mean \pm standard deviation. Comparisons: ${ }^{*}$ scalp region vs abdomen region $(p<0.05)$; ${ }^{* *}$ scalp region vs abdomen and waist regions $(p$ $<0.001)$. One-way analysis of variance (ANOVA) and post hoc Tukey test statistical analyses were used. 


\section{Scalp-Bulb}

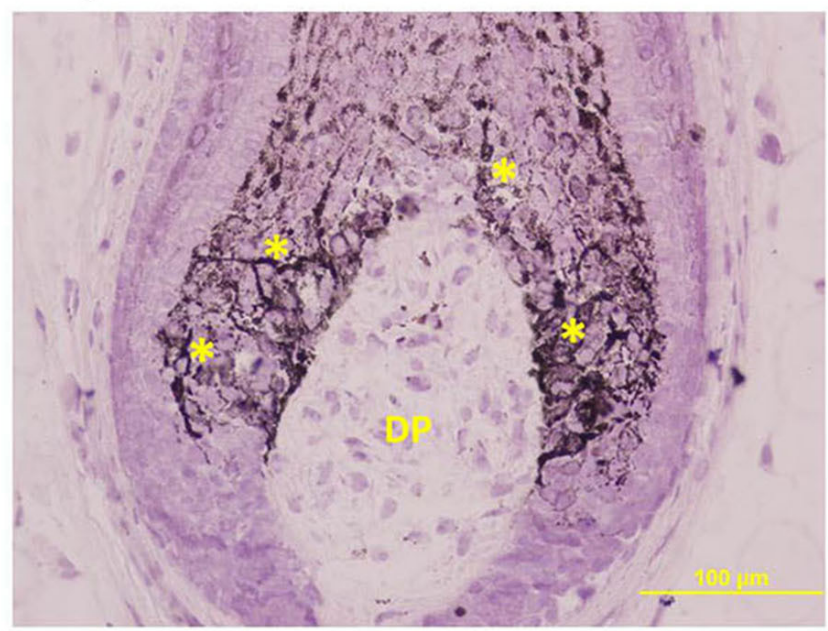

\section{Leg}

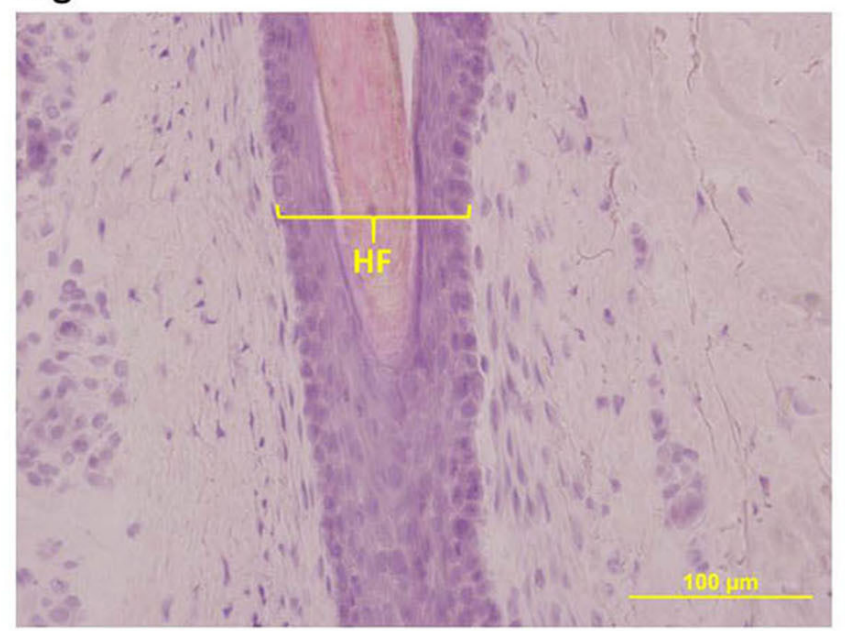

\section{Back}

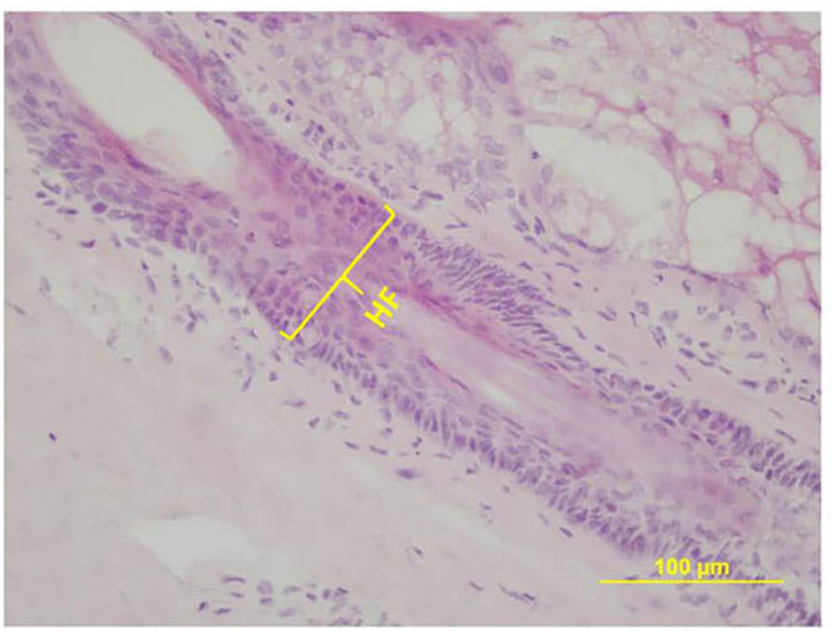

\section{Scalp-Bulge}

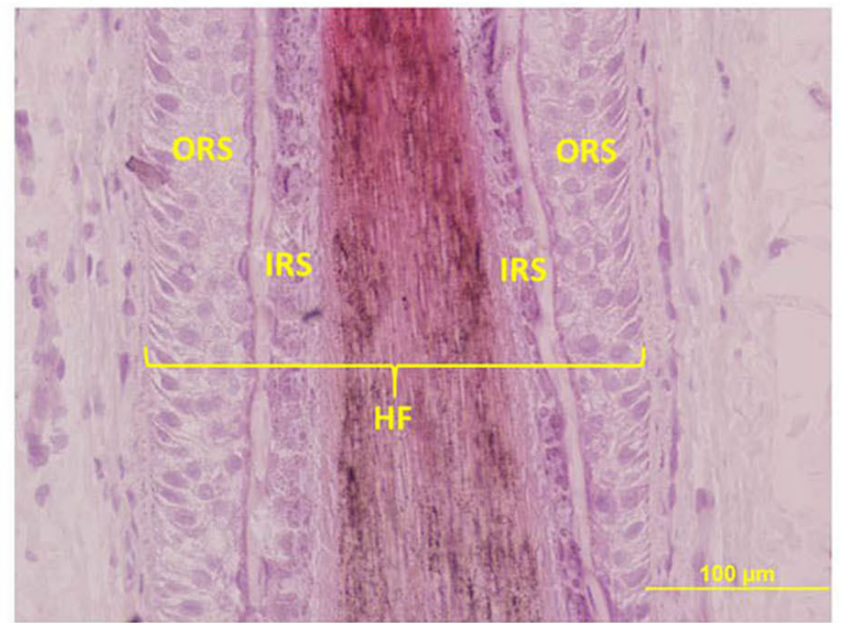

Abdomen

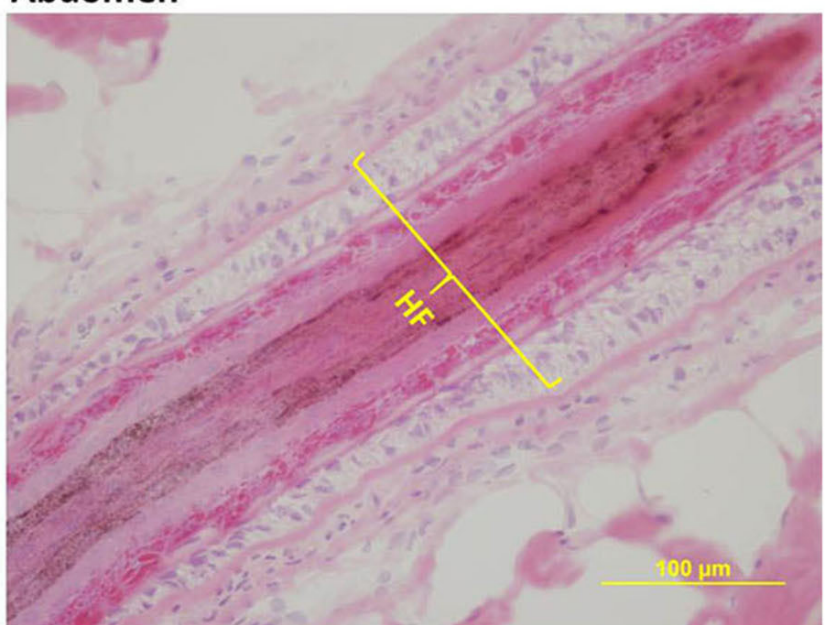

Waist

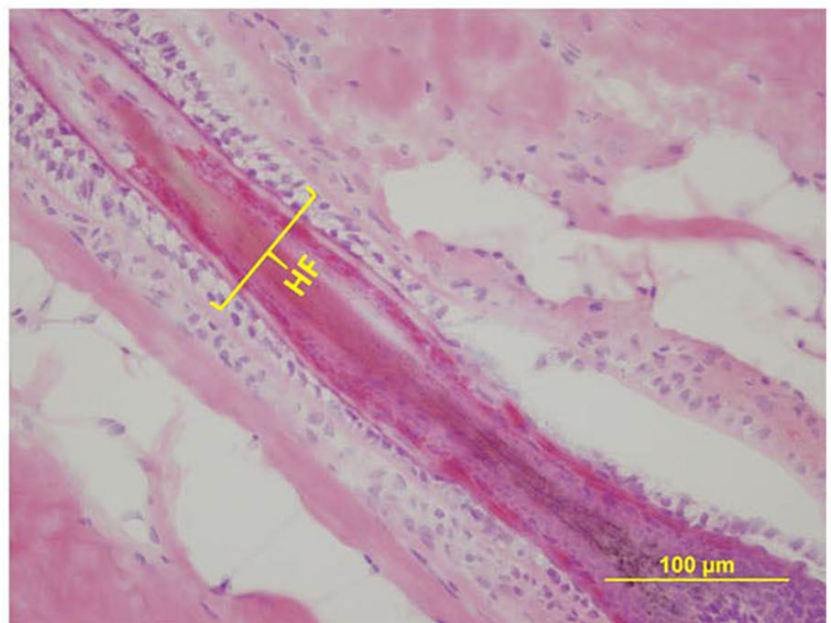

Fig. 1. Hematoxylin and eosin histochemistry: the overview of bulb and bulge regions of a HF of scalp tissue and the section of HFs taken from other skin regions

* melanocytes; DP - dermal papilla; HS - hair shaft; IRS - inner root sheath; ORS - outer root sheath; original magnification ( $\times 400$ ).

The cell apoptosis in the outer root sheath is most frequently assessed by the addition of synthetic, detectable nucleotides (dUTP) at DNA breaks by terminal transferase activity, that is TUNEL immunofluorescence, properly in conjunction with Ki-67 IR. It provides information to simultaneously show both proliferative and apoptotic cells by this method. Therefore, TUNEL and Ki67 documented massive intraepithelial apoptosis and proliferation of keratinocytes in the bulge. In the Ki-67 and TUNEL double-staining, whereas TUNEL-positive 


\section{Scalp}
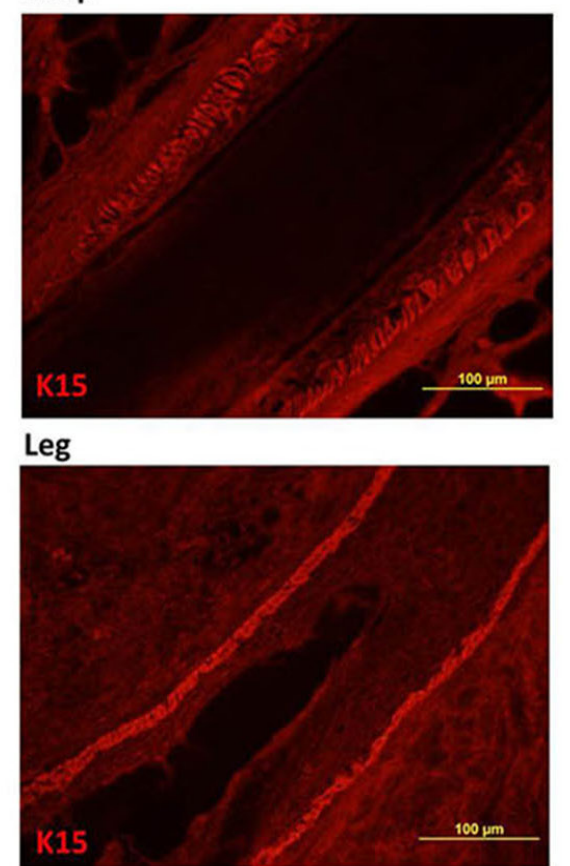

\section{Abdomen}

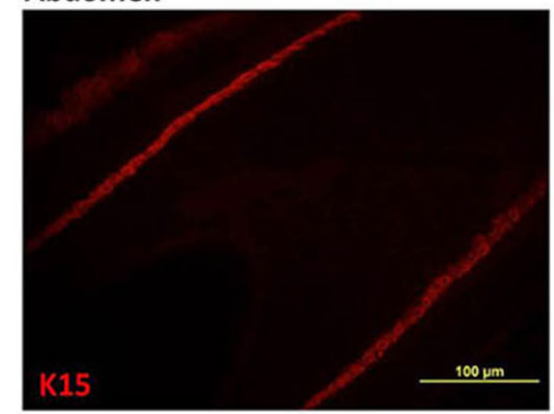

\section{Back}

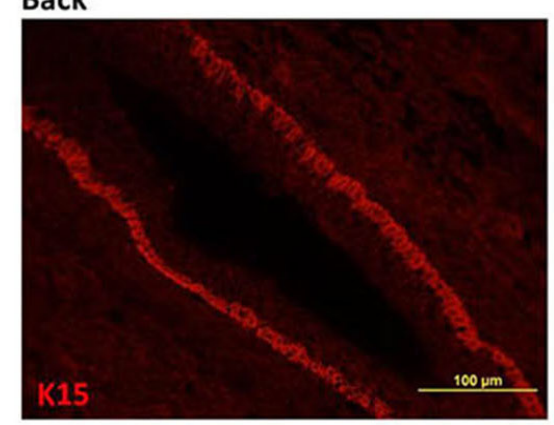

\section{Waist}

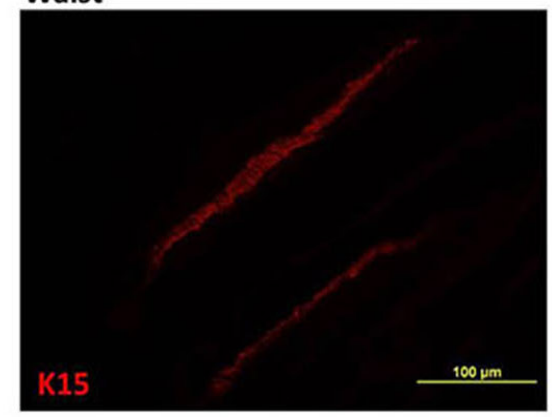

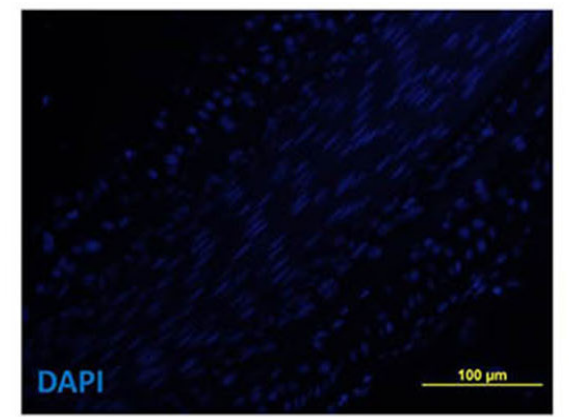
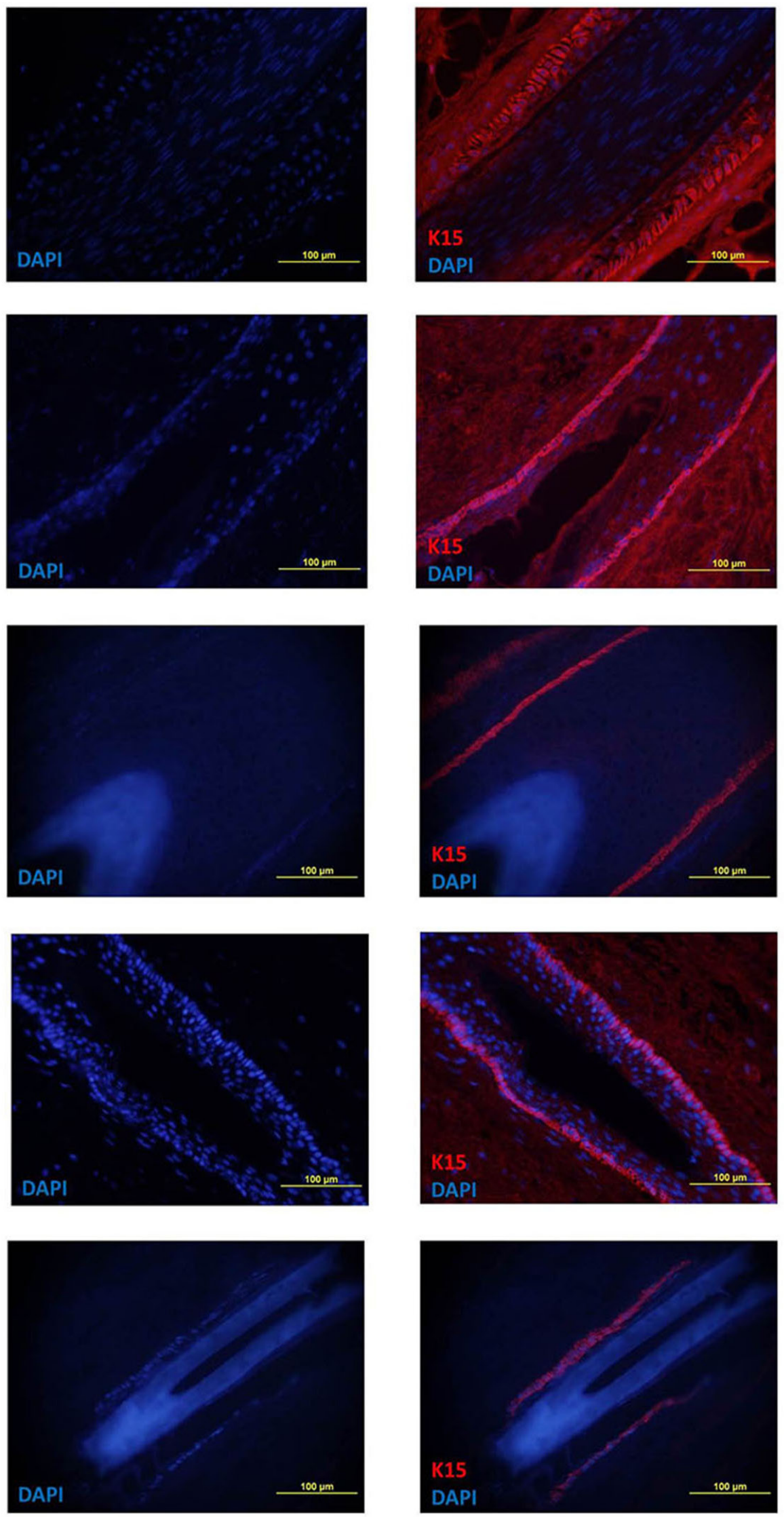

Fig. 2. The HF stem cells in the bulge region of the HFs from all different skin regions showing K15 IR. K15 immunofluorescence staining Original magnification (×400); DAPI - 4;6-diamidino-2-phenylindole. 
Scalp
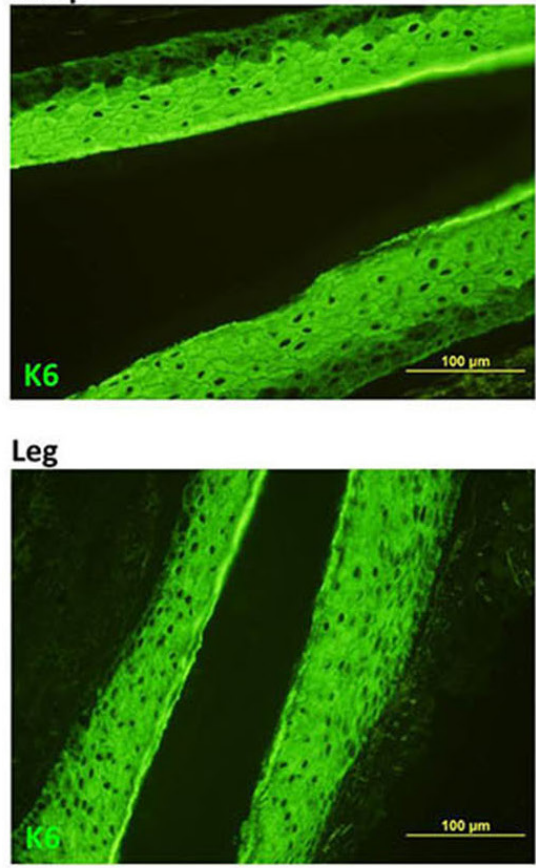

\section{Abdomen}
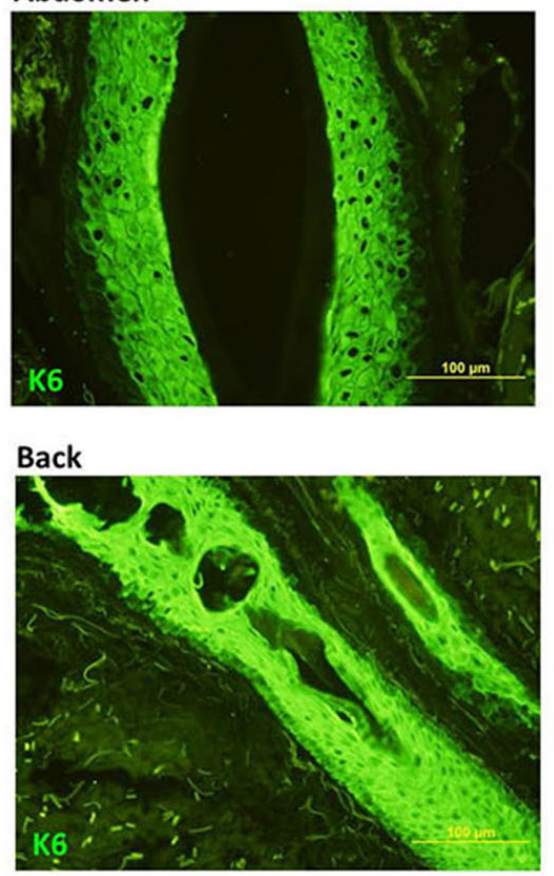

Waist

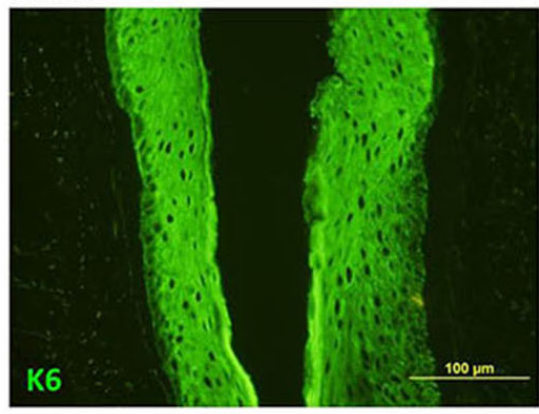

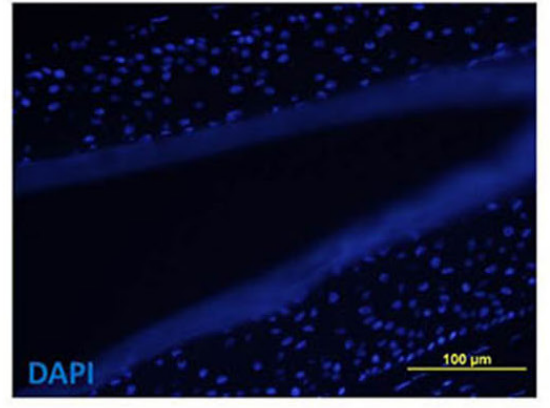
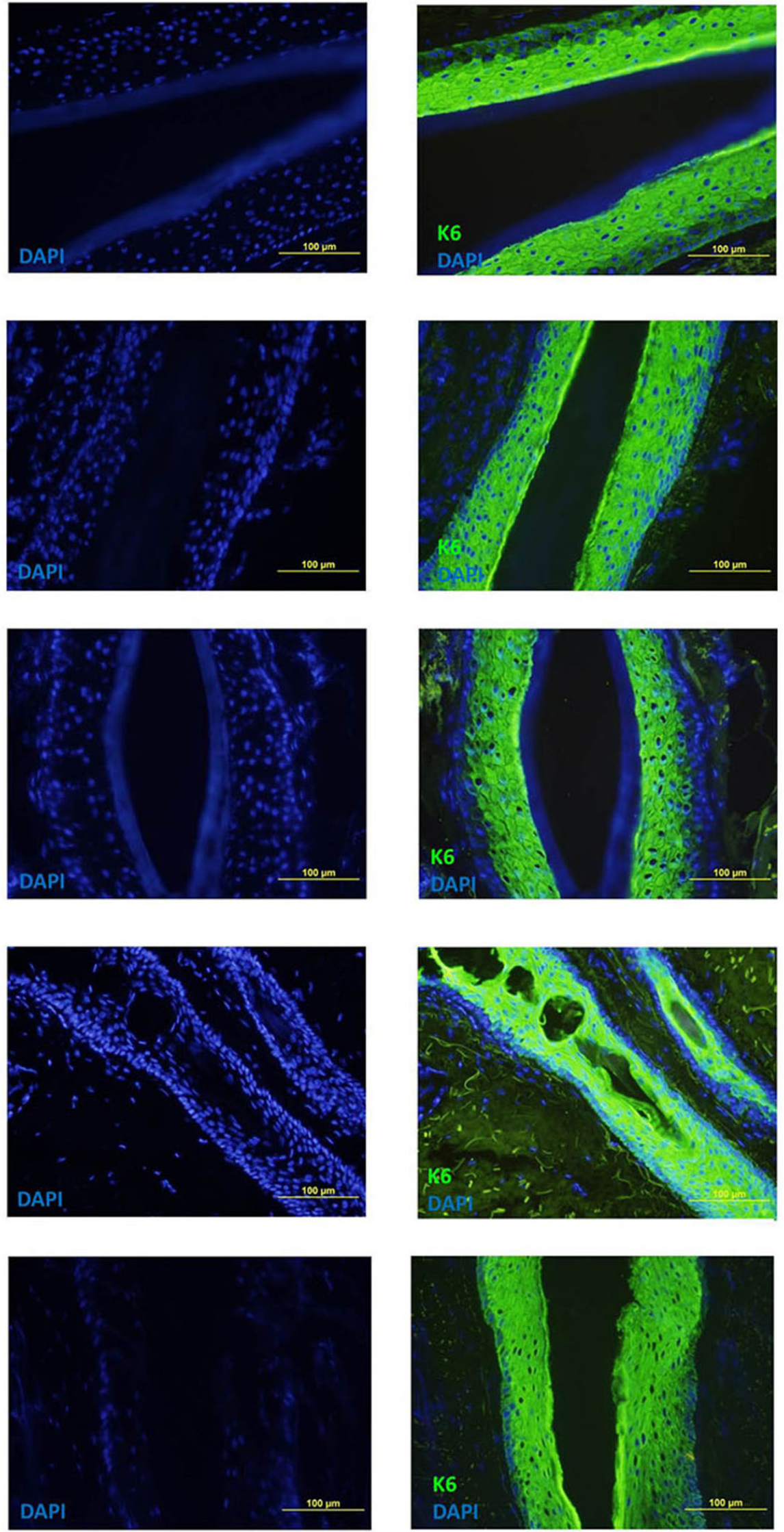

Fig. 3. Immunoreactivity intensity of K6 in the HFs of different body regions. K6 immunofluorescence staining Original magnification (×400); DAPI - 4;6-diamidino-2-phenylindole. 
cells seemed bright green, Ki-67-positive cells had bright red nuclei. The number of Ki-67-positive cells was highest in HFs of the scalp region, whereas the lowest Ki-67-positive cell number was observed in HFs of the abdomen region. However, this difference was not statistically significant ( $p>0.05)$. The number of TUNEL-positive cells was the highest in the HFs of scalp tissue, compared to the bulge regions of HFs obtained from other regions. There were TUNEL-positive cells in the bulge of HFs from different skin regions, but no significant difference between the scalp region and other regions in terms of the TUNELpositive cell numbers ( $p>0.05$ ) (Table 2, Fig. 4).

\section{Discussion}

Cytokeratins play a critical role in differentiation and tissue specialization, and function to maintain the overall structural integrity of epithelial cells. They are useful markers of tissue differentiation and also define the anatomical location of the niche, especially K15. Previous studies of the IR pattern of human HFSCs indicate that K15 IR is upregulated in the scalp human bulge compared with other regions of the ORS. The HFSCs were shown by immunostaining and microarray analyses, which demonstrated K15 upregulation in mice and human HF bulge cells. ${ }^{14}$ Under normal conditions, the epithelial compartments of the HF and interfollicular epidermis remain discrete, with K15-positive and bulge stem cells contributing progeny for HF reconstruction during the hair cycle. Garcin et al. have suggested that bulge cells only respond to epidermal wounding during later stages of repair. ${ }^{25}$ In our study, we investigated the outer root sheath of human HFs taken from 5 different skin regions, the head, leg, abdomen, back and waist, by using the K15 marker. We demonstrated that the highest expression was in the HFSCs taken from the scalp tissue. Furthermore, the HFSCs in the back and leg region showed nearly the same HFSCs as in the scalp, as opposed to in the waist and abdominal regions. This finding suggests that these regions may be as suitable as HFs taken from scalp tissue for stem cell isolation.

Additionally, suprabasal expression of K15 has also been reported in both normal and diseased tissues, which is incompatible with its role as a stem cell marker. ${ }^{26}$ Many disorders of the skin, such as cancer, chronic wounds, skin atrophy and fragility, hirsutism, and alopecia, can be investigated as disorders of adult stem cells. Because stem cells in the epidermis and HF serve as an excellent source of cells, understanding the control of their proliferation and differentiation is crucial to fully understand the disorders associated with the disruption in these processes. ${ }^{27}$ K15 has been indicated to be expressed in variants of human basal cell carcinoma and other neoplasms of skin appendages such as sebaceous neoplasms and trichoepithelioma. ${ }^{28}$ A study by Quist et al. showed that expression of potential stem cell markers of the epidermis and
HF was observed in the skin tumors of appendages and human basal cell carcinomas. ${ }^{29}$ Also, they demonstrated that many of these markers appeared to be downregulated during tumor progression. These studies have expressed the importance of general HF markers in that it can change expression levels in some pathological conditions. Therefore, it is important to determine the expression levels of specific markers in HFs taken from different skin regions. Although there are numerous immunohistochemical and immunofluorescence studies of HF specific markers on both normal and pathologic skin, expression patterns in the HFs of the different skin regions has been not been addressed. Our study is the $1^{\text {st }}$ one to investigate this hypothesis. We investigated the expression patterns of K15, K6 and proliferative cell number with Ki-67 in different skin regions. In the present study, the HFSCs showed K15-positive expression in all of the HFs taken from different skin regions in the ORS of the follicle. According to our results, K15 IR intensity was higher in the HFSCs of the scalp tissue than in other tissues.

It may be considered that the role of constitutive $\mathrm{K} 6$ expression is one reinforcement needed only to withstand increased mechanical stress. It is a marker for the proliferating epithelial layer and is exudative in keratinocytes in the specialized epithelium of the HF. In the current study, the HF keratinocytes taken from different skin regions were determined by $\mathrm{K} 6$ immunofluorescent staining method and K6 IR was present in all HFs taken from different skin regions, especially the innermost cells surrounding the hair shaft as is supported by other studies. ${ }^{2,16}$ Some lesions reflect a loss of the structural support function shared by $\mathrm{K} 6$, other keratins or intermediate filament proteins. In our study, most of the $\mathrm{K} 6$ expression was observed in the HFs belonging to the scalp tissue, whereas the HFs of the back region showed a $\mathrm{K} 6$ expression density close to scalp tissue. Additionally, in the HF sections of the other regions (leg, abdomen, and waist) there was less expression intensity of $\mathrm{K} 6$ than the densities of expression in the back and scalp region, but these results were not statistically significantly different.

The proliferative and apoptotic cell numbers of the HFSCs in different skin regions are also important, considering the studies on HFSCs. Ki-67, a cell proliferation marker, is a useful component for gaining information on cell proliferation in cryosections and is commonly used in HF studies. During anagen, Ki-67 IR is most prominent in hair matrix keratinocytes below the widest part of the dermal papilla. The onset of catagen is described by a marked reduction in the percentage of Ki-67-positive matrix keratinocytes, with virtually no Ki-67-positive HF keratinocytes present in late catagen. ${ }^{18}$ Contrary to conventional information, even in telogen HFs, some keratinocytes still enter into the cell cycle. ${ }^{30}$ In our study, we performed Ki-67 and TUNEL double staining to show cell proliferation and apoptosis. The number of Ki-67-positive cells was observed in keratinocytes 


\section{Abdomen}

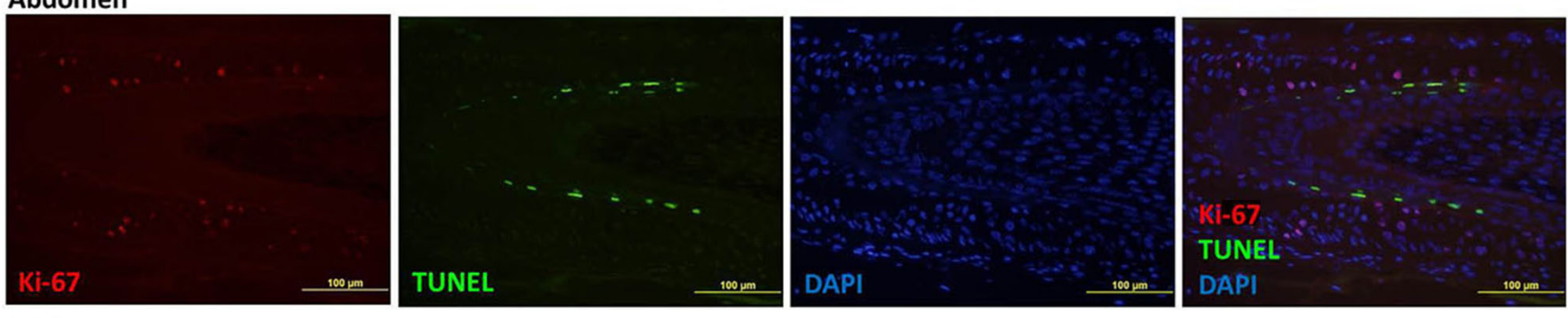

Back
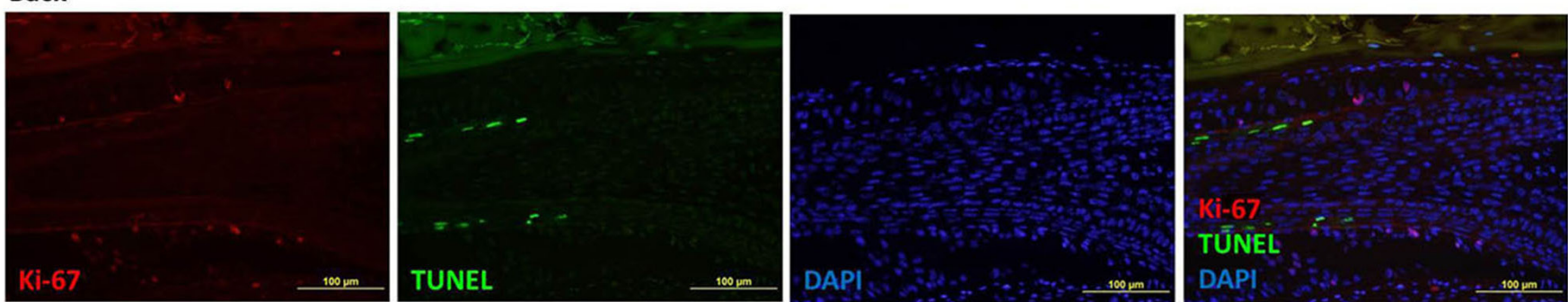

\section{Abdomen}
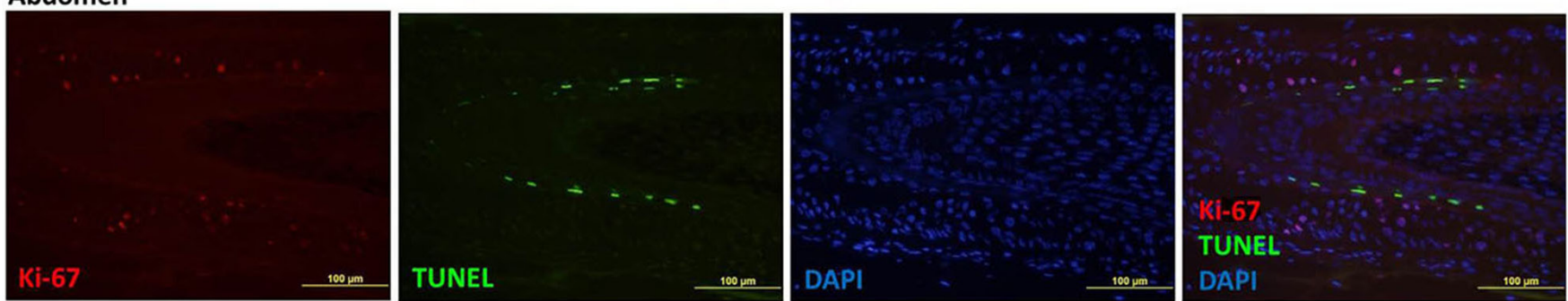

Back
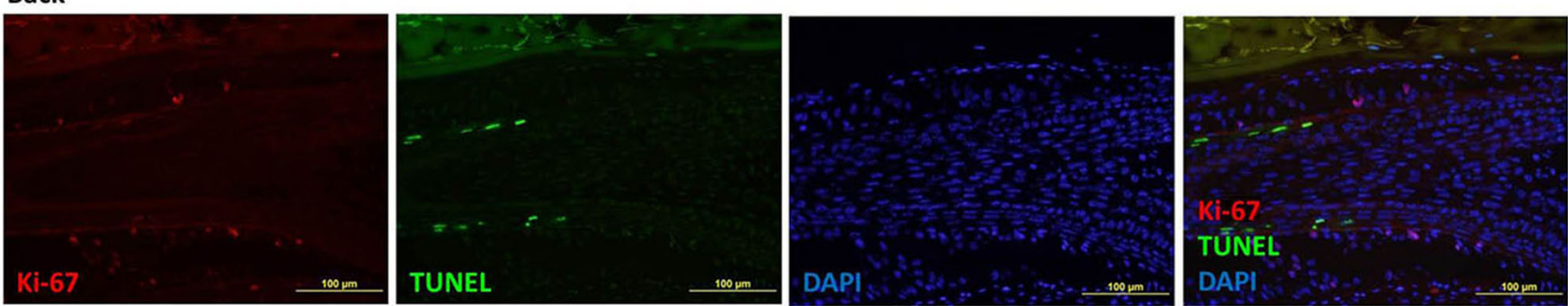

\section{Waist}
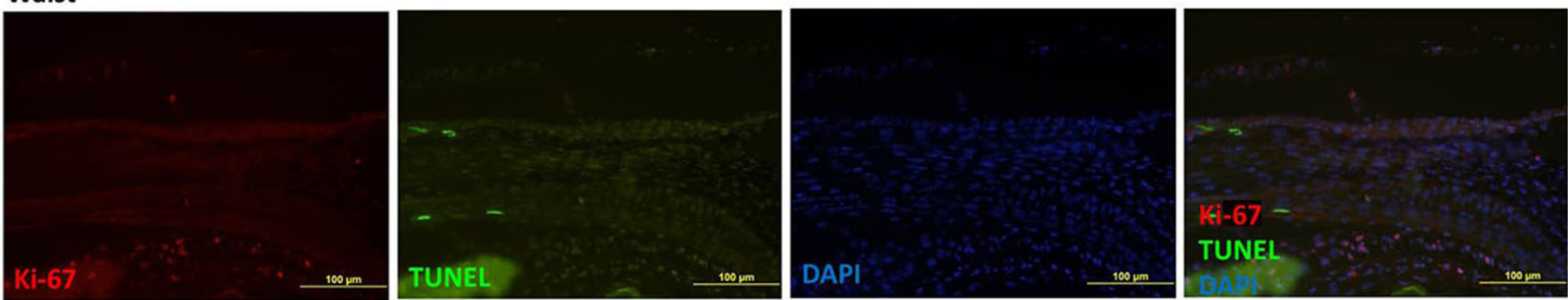

Fig. 4. While apoptotic cells were labeled with TUNEL, proliferative cells were determined with Ki-67 in ORS keratinocytes of HF bulges from different skin regions. TUNEL-positive cells reflect green immunofluorescence, whereas Ki-67-positive cells reflect red immunofluorescence

TUNEL - terminal deoxynucleotidyl transferase-mediated digoxigenin-dNTP nick end-labelling; ORS - outer root sheath; DAPI - 4;6-diamidino-2-phenylindole.

in the bulge of all HFs taken from different skin regions. Additionally, apoptosis was also viewed in some cells that form the HF bulge of different skin regions. In this study, there was no significant difference in the Ki-67 and TUNEL-positive cell numbers in the keratinocytes of the HF bulge regions taken from different skin regions.

In recent years, cell-based therapies, particularly those using stem cells, for improving survival, have emerged as a promising approach for the treatment of severe diseases. The conversion of stem cells to other cells has also increased the interest in these cells. The ease of tissue harvesting and multipotent nature of the resident stem cells in the HF has encouraged basic and clinical research in this area. As a novel cell source, mesenchymal stem cells separated from human HFs are appropriate to obtain, have no age limit, and are easily accessible. ${ }^{31}$ These results have 
increased the interest especially in working on HFSCs. However, it may also be important to know which skin regions the HF should be taken from in such studies.

There have been many studies on the size and diameter of the HF and also the volume of the dermal papillae in different body regions. ${ }^{22,32,33}$ Otberg et al. showed the diversity of HF sizes in different body regions in their study, which emphasizes the importance of understanding and calculating the follicular penetration and permeability processes of HF density and size. ${ }^{22}$ Only a few studies have been performed for the determination of vellus $\mathrm{HF}$ density on the human body. ${ }^{11,22}$ A study by Blume et al. determined the HF density on the forehead, cheek, chest, and back. ${ }^{32}$ In this study, an average density of 423 follicles per $\mathrm{cm}^{2}$ was identified on the forehead and a mean density of 92 follicles on the back. Additionally, Pagnoni et al. found a density of 455 HFs on the lateral forehead and the highest density on the nasal wing, with 1220 follicles per $\mathrm{cm}^{2} .{ }^{33}$ However, because of the relatively lower growth of the head in comparison to the extremities, the HFs are much more numerous on the face and scalp than on the legs and arms. ${ }^{33}$ On the other hand, Otberg et al. stated that the highest HF density and percentage of follicular orifices on the skin and infundibular surfaces were observed on the forehead, whereas the highest average size of the follicular orifices was measured in the calf region. ${ }^{22}$ In their study, the highest infundibular volume, and therefore a potential follicular reservoir, was calculated for the forehead and the calf region, although the calf region showed the lowest HF density.

Studies on the functions, molecular mechanisms and hormonal controls of HFSCs continue. In the research literature, there were no studies on the expression of HF specific markers in vellus type HFs in different regions of the human body as well as terminal HFs. Therefore, the knowledge of IR intensity of specific markers in the HFs obtained from different areas can be important for understanding the characteristics of the isolated HF. This was a preliminary study that investigated the IR of specific markers in the HFs according to different skin regions. Here, we have shown that the IR of HF specific markers can vary according to different skin regions. These findings might play a crucial role for tissue sample diagnosis and prognosis of being both biologically and clinically important in the future, and could be exploited for research and therapeutic purposes. Further studies are required to address whether the differences in the expression patterns of molecular markers is a result of an age-related dissociation or a site-specific differentiation at the tissue and cellular levels in humans.

\section{References}

1. Niemann C, Watt FM. Designer skin: Lineage commitment in postnatal epidermis. Trends Cell Biol. 2002;12:185-192.

2. Blanpain C, Lowry WE, Geoghegan A, et al. Self-renewal, multipotency, and the existence of two cell populations within an epithelial stem cell niche. Cell. 2004;118:635-648.
3. Langbein $L$, Rogers MA, Winter $H$, et al. The catalog of human hair keratins. II. Expression of the six type II members in the hair follicle and the combined catalog of human type I and II keratins. J Biol Chem. 2001;276:35123-35132.

4. Magin TM, Vijayaraj P, Leube RE. Structural and regulatory functions of keratins. Exp Cell Res. 2007;313:2021-2032.

5. Moll R, Divo M, Langbein L. The human keratins: Biology and pathology. Histochem Cell Biol. 2008;129:705-733.

6. Langbein L, Schweizer J. Keratins of the human hair follicle. Int Rev Cytol. 2005;243:1-78.

7. Schweizer J, Bowden PE, Coulombe PA, et al. New consensus nomenclature for mammalian keratins. J Cell Biol. 2006;174:169-174.

8. Troy TC, Arabzadeh A, Turksen K. Re-assessing K15 as an epidermal stem cell marker. Stem Cell Rev. 2011;7(4):927-934.

9. Liu Y, Lyle S, Yang Z, et al. Keratin 15 promoter targets putative epithelial stem cells in the hair follicle bulge. J Invest Dermatol. 2003;121:963-968.

10. Webb A, Li A, Kaur P. Location and phenotype of human adult keratinocyte stem cells of the skin. Differentiation. 2004;72:387-395.

11. Pontiggia L, Biedermann T, Meuli M, et al. Markers to evaluate the quality and self-renewing potential of engineered human skin substitutes in vitro and after transplantation. J Invest Dermatol. 2009;129:480-490.

12. Roh C, Roche M, Guo Z, et al. Multi-potentiality of a new immortalized epithelial stem cell line derived from human hair follicles. In Vitro Cell Dev Biol Anim. 2008;44:236-244.

13. Blanpain C, Fuchs E. Epidermal homeostasis: A balancing act of stem cells in the skin. Nat Rev Mol Cell Biol. 2009;10:207-217.

14. Ohyama M, Terunuma A, Tock CL, et al. Characterization and isolation of stem cell-enriched human hair follicle bulge cells. J Clin Invest. 2006;116:249-260.

15. Omoto M, Miyashita $H$, Shimmura S, et al. The use of human mesenchymal stem cell-derived feeder cells for the cultivation of transplantable epithelial sheets. Invest Ophthalmol Vis Sci. 2009;50:2109-2115.

16. Hsu YC, Pasolli HA, Fuchs E. Dynamics between stem cells, niche, and progeny in the hair follicle. Cell. 2011;144:92-105.

17. Scholzen T, Gerdes J. The Ki-67 protein: From the known and the unknown. J Cell Physiol. 2000;182:311-322.

18. Kloepper JE, Sugawara K, Al-Nuaimi Y, et al. Methods in hair research: How to objectively distinguish between anagen and catagen in human hair follicle organ culture. Exp Dermatol. 2010;19:305-312.

19. Chamcheu JC, Siddiqui IA, Syed DN, et al. Keratin gene mutations in disorders of human skin and its appendages. Arch Biochem Biophys. 2011;508:123-137.

20. Mohanty S, Kumar A, Dhawan J, et al. Noncultured extracted hair follicle outer root sheath cell suspension for transplantation in vitiligo. Br J Dermatol. 2011;164:1241-1246.

21. Harries MJ, Meyer KC, Chaudhry IH, et al. Does collapse of immune privilege in the hair-follicle bulge play a role in the pathogenesis of primary cicatricial alopecia? Clin Exp Dermatol. 2010;35:637-644.

22. Otberg $\mathrm{N}$, Richter $\mathrm{H}$, Schaefer $\mathrm{H}$, et al. Variations of hair follicle size and distribution in different body sites. J Invest Dermatol. 2004;122:14-19.

23. Kloepper JE, Tiede S, Brinckmann J, et al. Immunophenotyping of the human bulge region: The quest to define useful in situ markers for human epithelial hair follicle stem cells and their niche. Exp Dermatol. 2008;17:592-609.

24. Foitzik K, Hoting E, Förster T, et al. L-carnitine-L-tartrate promotes human hair growth in vitro. Exp Dermatol. 2007;16:936-945.

25. Garcin CL, Ansell DM, Headon DJ, et al. Hair follicle bulge stem cells appear dispensable for the acute phase of wound re-epithelialisation. Stem Cells. 2016;34:1377-1385.

26. Bose A, Teh MT, Mackenzie IC, et al. Keratin K15 as a biomarker of epidermal stem cells. Int J Mol Sci. 2013;14:19385-19398.

27. Stenn KS, Cotsarelis G. Bioengineering the hair follicle: Fringe benefits of stem cell technology. Curr Opin Biotechnol. 2005;16:493-497.

28. Evangelista MT, North JP. Comparative analysis of cytokeratin 15, TDAG51, cytokeratin 20 and androgen receptor in sclerosing adnexal neoplasms and variants of basal cell carcinoma. J Cutan Pathol. 2015;42:824-831.

29. Quist SR, Eckardt M, Kriesche A, et al. Expression of epidermal stem cell markers in skin and adnexal malignancies. Br J Dermatol. 2016;175:520-530. 
30. Geyfman M, Plikus MV, Treffeisen E, et al. Resting no more: Re-defining telogen, the maintenance stage of the hair growth cycle. Biol Rev Camb Philos Soc. 2015;90:1179-1196.

31. Xu S, Shuang L, Xia H, et al. Differentiation of hepatocytes from induced pluripotent stem cells derived from human hair follicle mesenchymal stem cells. Cell Tissue Res. 2016;366:89-99.

32. Blume U, Ferracin J, Verschoore M, et al. Physiology of the vellus hair follicle. Hair growth and sebum excretion. Br J Dermatol. 1991;124:21-28.

33. Pagnoni AP, Kligman AM, Gammal SEL, et al. Determination of density of follicles on various regions of the face by cyanoacrylate biopsy: Correlation with sebum output. Br J Dermatol. 1994;131:862-865. 